\title{
Digital intraosseous epidermoid inclusion cyst of the distal phalanx
}

\author{
David E. Ruchelsman · Daniel K. Laino · Kimberlly S. Chhor · German C. Steiner · Samuel Kenan
}

Received: 17 January 2009 / Accepted: 7 February 2009

(C) Society of Hand and Microsurgeons of India 2010

\begin{abstract}
Epidermoid inclusion cysts (EIC) of the bone are exceedingly rare. We present a case of an atypical EIC originating at the base of the distal phalanx of the index finger following a remote history of crush injury to the finger. The differential diagnosis of expansile, lytic lesions of the phalanges remains broad, and definitive diagnosis requires tissue histopathological analysis. At latest follow-up, the patient was pain-free and obtained an excellent clinical and radiographic outcome following intra-lesional curettage and bone grafting. Differentiation of EIC from other radiolucent digital lesions remains challenging, especially when classic radiographic findings are not seen. We review the clinical, radiographic, and pathologic diagnostic features of this lesion, as well as our current treatment algorithm.
\end{abstract}

Keywords Epidermoid inclusion cyst · Phalanx lesion . Hand tumor

David E. Ruchelsman ${ }^{1}(\varangle) \cdot$ Daniel K. Laino ${ }^{1} \cdot$ Kimberlly S. Chhor ${ }^{1} \cdot$ German C. Steiner ${ }^{2} \cdot$ Samuel Kenan ${ }^{1}$

${ }^{1}$ Department of Orthopaedic Surgery

${ }^{2}$ Department of Musculoskeletal Pathology

New York University Hospital for Joint Diseases

New York, USA

Investigation performed at New York University Hospital for Joint Diseases, New York, USA

e-mail: david.ruchelsman@nyumc.org

\section{Introduction}

Digital distal phalangeal lesions remain uncommon entities, and the diagnostic considerations broad. Intraosseous epidermoid inclusion cyst is an exceedingly rare entity and may be the result of remote digital trauma given the unique regional anatomy of the terminal phalanx. Only limited case reports of epidermoid inclusion cysts of bone appear in the orthopaedic literature. We present a case of this rare pathological entity in the distal phalanx of the index finger. Furthermore, this lesion was located in an atypical location along the longitudinal axis of the terminal phalanx. We review our diagnostic and treatment algorithm of this rare intraosseous digital lesion.

\section{Case report}

A 26-year-old right-hand dominant African American female without significant medical history presented to our tertiary orthopaedic oncology clinic with complaints of recurrent pain localized to the tip of her dominant index finger for approximately five months. Five weeks prior to presentation, she had sustained a fracture of the distal phalanx without antecedent trauma and was treated with immobilization. Her musculoskeletal history was significant for a remote history of a crush injury to the same finger approximately 12 years ago.

Examination of the index finger revealed minimal swelling with circumferential tenderness about the distal phalanx of the index finger without an associated soft tissue mass. There was full, painless active and passive motion of the distal interphalangeal joint and a full total arc of motion of the digit.

Preoperative radiographs (Fig. 1) demonstrated a wellcircumscribed, lytic, expansile lesion originating at the base of the distal phalanx along the subarticular surface. Cortical thinning was evident without pathologic fracture. Preoperative MRI (Fig. 2) revealed an expansile, lytic 
lesion the distal phalanx with cortical disruption. Preoperative imaging suggested a benign cartilage tumor (enchondroma).

Given the clinical and radiographic findings, the patient was indicated for operative intervention. Intralesional curettage and demineralized cancellous bone grafting were performed taking care to preserve the articular surface of the distal phalanx. Histopathological analysis (Fig. 3) demonstrated the presence of stratified squamous cell epithelium with prominent keratinization, consistent with a diagnosis of an intraosseous epidermal inclusion cyst. There was no evidence of chondrocytes or cartilaginous tissue to support a diagnosis of enchondroma.

At 8 months postoperatively, the patient was pain-free. Clinical examination demonstrated full DIP and digital total arc of motion. Radiographs at latest follow-up revealed complete osseous healing with incorporation of the bone graft within the distal phalanx and preservation of the articular surface (Fig. 4).

\section{Discussion}

The differential diagnoses of phalangeal lesions are broad, and include inflammatory (i.e. chronic infection), as well as benign and malignant processes. Diagnostic considerations are dependent upon the location of the lesion along the longitudinal axis of the phalanx. Proximally-based lesions are most commonly enchondromas (benign cartilage tumor) or intraosseous synovial cysts. Distally-based erosive, cystic lesions include those secondary to tophaceous gout or a localized giant cell tumor of tendon sheath, giant cell reparative granuloma, simple bone cyst, aneurysmal bone cyst, osteoid osteoma, and epidermoid inclu-
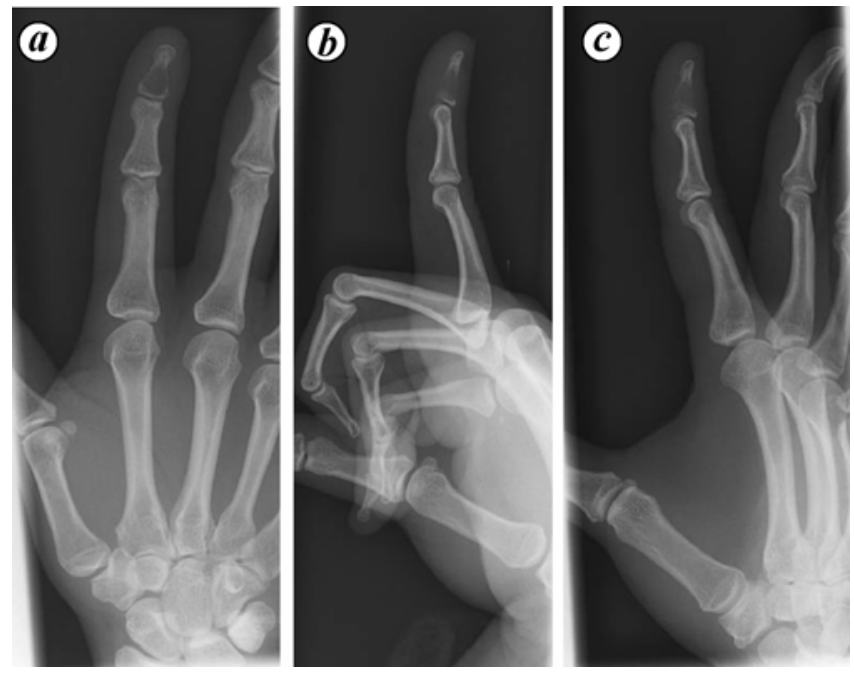

Fig. 1. Preoperative anteroposterior (a), lateral (b), and oblique (c) radiographs demonstrated a lytic, expansile lesion originating proximally at the subchondral base of the distal phalanx with distal extension sion cyst. Primary malignant tumors are exceedingly rare in the distal phalanges. In the appropriate clinical setting, metastatic lesions remain a diagnostic consideration. The most common malignant tumor to be seen in the distal phalanx is metastatic lung carcinoma.

Epidermal inclusion cysts (EIC) of the bone remain a rare entity. These lesions occur most frequently in the distal phalanges of the hand and in the skull $[1-3,5-6]$, but have been reported in the toes as well $[4,7]$. In a review of 10 cases of intraosseous epidermal inclusion cysts (distal phalanges of the hand, $n=9$; toe, $n=1$ ), Roth [5] observed that pain was the most common presenting symptom. Less common symptoms include increasing finger size and nail deformity [6]. The average age of presentation is approximately $30-50[5,6]$, but cases have been reported in children as young as 8 years old [3]. The lesions appear to occur more frequently in men than women $[5,6]$.

The etiology of intraosseous epidermal inclusion cysts remains unknown. While a congenital etiology with intraosseous inclusion of embryonal epithelial tissue has been proposed [6], a post-traumatic origin remains the most prevalent hypothesis as many patients do recall an antecedent traumatic event (such as crush injury or amputation [6]) or surgical procedure in the area of the identified lesion. It is surmised that superficial epithelial cells are displaced into the underlying osseous compartment following digital trauma or surgery. EIC most commonly are seen in the distal phalanges and this phenomenon is thought to be secondary to the unique regional anatomy as the subungual bed adheres to the underlying periosteum [6]. Symptoms may present at a variable amount of time (i.e. months to years) following the inciting event [4-7]. We emphasize that in this case, it was only after further examination of the history following return of the pathology results, that the patient endorsed a history of a crush injury to the distal tip of the right index finger twelve years ago.

Radiographically, intraosseous EIC typically presents as a well-defined osteolytic lesion without any trabecular pattern. There may be cortical thinning or erosion, and typically bony sclerosis or reaction is absent [1, 4-7]. Radiographically, these lesions are typically based at the most distal aspect of the distal phalanx (Fig. 5). Enchondroma remains the most common bone tumor of the hands and feet and is the most frequent lesion seen in the terminal phalanx of the digits [6]. Like EIC, enchondromas are osteolytic lesions, but often display intralesional calcification. Further, enchondromas classically involve the proximal aspect of the distal phalanx (Fig. 6), and EIC is typically confined to the distal aspect [6]. We highlight that in this case the lesion originated at the proximal aspect of the distal phalanx along the subchondral surface. Its localization to the proximal one-half of the longitudinal axis of the distal phalanx (Figs 1,2) was atypical. Had this lesion originated radio- 

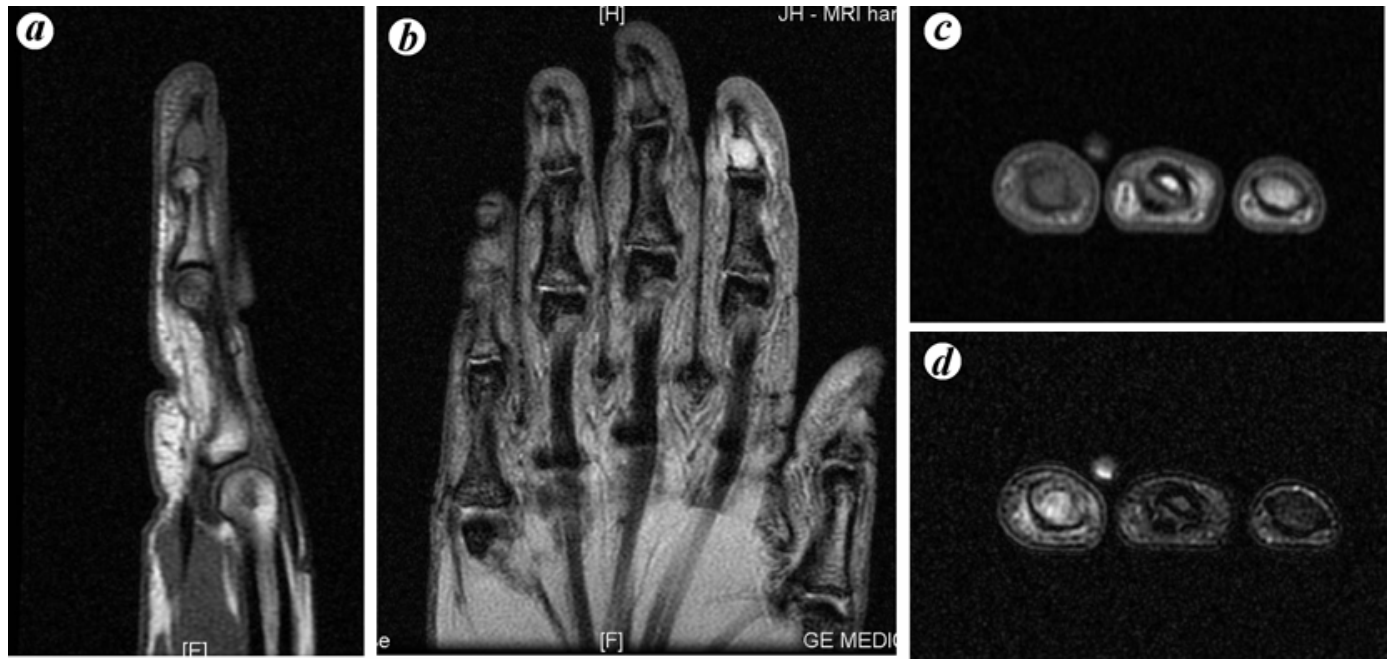

Fig. 2. Preoperative MRI sequences: Sagittal T1 (a) images demonstrate a low signal lesion within the proximal portion of the distal phalanx; corresponding coronal (b) image shows the regional high signal lesion; the post contrast T1 axial image (c) reveals a peripherally enhancing lesion, and the corresponding fat saturation $\mathrm{T} 2$ axial (d) shows soft tissue edema
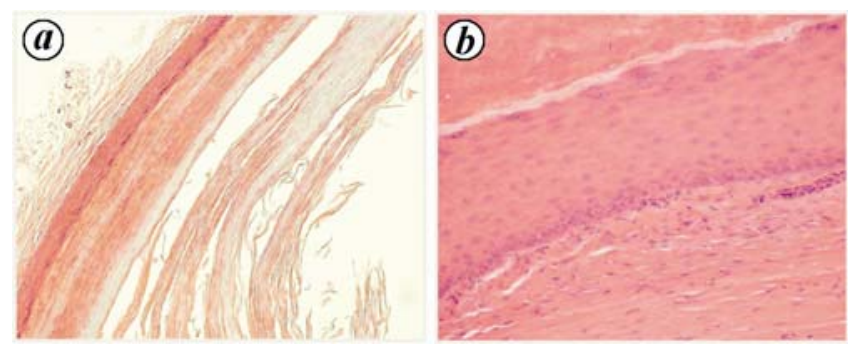

Fig. 3. Low power view (a) of the cyst wall showing a thin layer of squamous cell epithelium and abundant keritanization. High power view (b) of the keratinized squamous cell epithelium
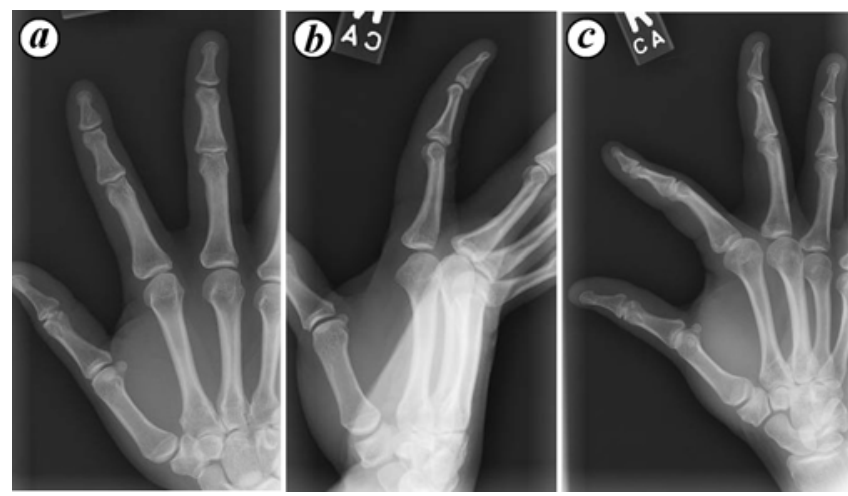

Fig. 4. Latest postoperative anteroposterior (a), lateral (b), and oblique (c) radiographs demonstrates full osseous healing with incorporation of the bone graft and preservation of the articular surface

graphically within the distal aspect of the distal phalanx, diagnostic consideration preoperatively would have included EIC of bone. Additionally, as discussed above, the Springer

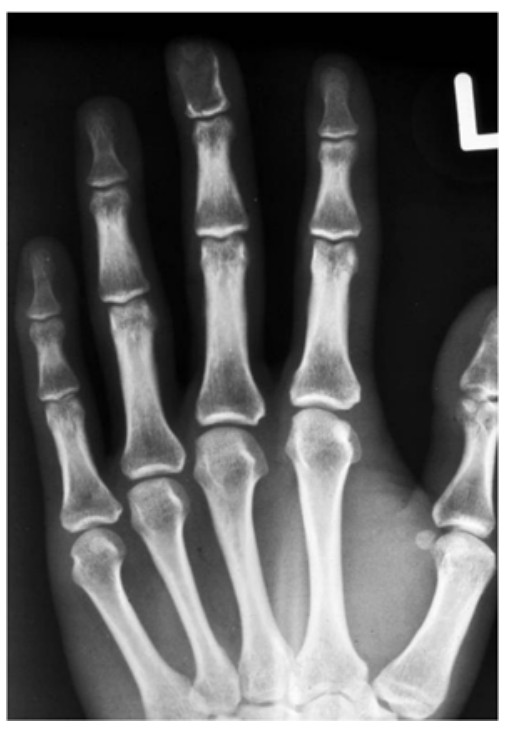

Fig. 5. Typical EIC of the middle finger. The lesion originated within the distal segment of the phalanx, and with time, progressed to involve almost the entire medullary compartment of the distal phalanx.

patient only endorsed the remote history of digital crush injury upon postoperative follow-up once a diagnosis of EIC of bone was made.

Radiographic distinction between distal phalangeal lesions will continue to remain challenging, especially when atypical radiographic features are seen. Thus, definitive diagnosis requires tissue analysis obtained during curettage. Less often, fine needle aspiration can also be used to make the diagnosis [2]. EIC lesions are typically one to two centimeters in diameter and filled with yellowish-white cheesy material. Histologically, they are 

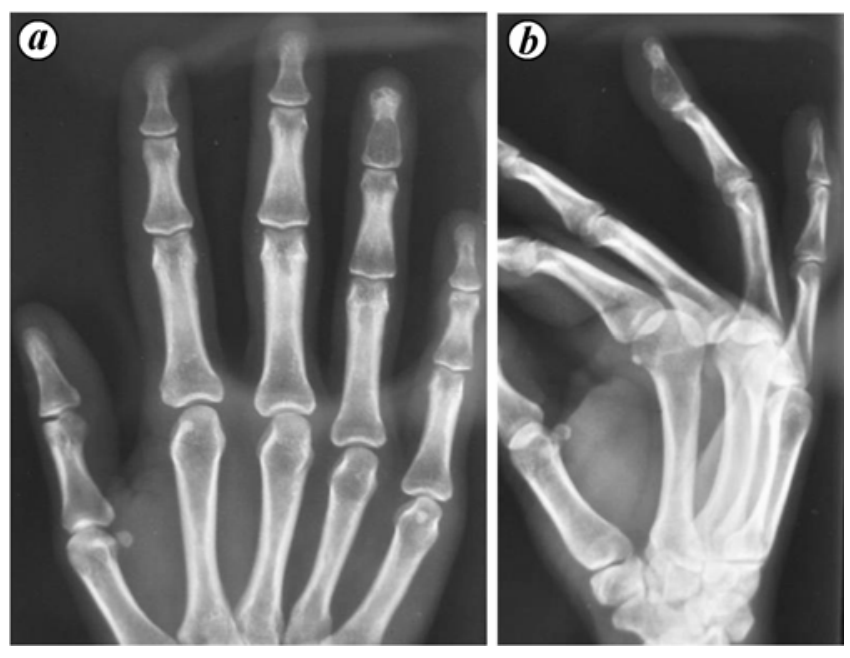

Fig. 6. Anteroposterior (a) and lateral (b) radiographs of an enchondroma of the ring finger located in the proximal segment of the phalanx. Note the similar radiographic features of the EIC in Fig. 1

separated from the surrounding bone by a layer of connective tissue. The cysts are lined with stratified squamous epithelium with a prominent granular layer and filled with abundant keritan debris [4-7]. Typically no skin appendages are seen [5-7].

The surgical treatment of EIC consists of intra-lesional curettage with or without bone graft $[1,3,4-5]$. Uneventful recovery with no evidence of recurrence at short-term follow-up [1,3-4] may be anticipated. Based on the limited published literature, there have been no reported cases of recurrence following curettage.

\section{Summary}

Intraosseous epidermoid cyst of the distal phalanx remains a relatively rare pathologic entity. It must be dif- ferentiated from other radiolucent phalangeal lesions. A remote or recent history of digital trauma should be sought in order to narrow the diagnostic possibilities. Intraosseous epidermoid inclusion cyst is difficult to differentiate radiographically from other radiolucent lesions and tissue diagnosis is required. We emphasize based on this case that these lesions may not always originate from or be confined to the most distal aspect of the distal phalanx. Therefore, a history of digital trauma should be sought in any patient presenting with a distal phalanx lesion present anywhere along the longitudinal axis of the phalanx. Intra-lesional curettage and bone grafting has yielded successful clinical and radiographic results in the limited number of cases reported to date.

\section{References}

1. Hamad AT, Kumar A, Anand Kumar C (2006) Intraosseous epidermoid cyst of the finger phalanx: a case report. J Orthop Surg 14:340-342

2. Handa U, Kumar S, Mohan H (2002) Aspiration cytology of epidermoid cyst of the terminal phalynx. Diagn Cylopathol 26:266-267

3. Hensley CD (1966) Epidermoid cyst of the distal phalynx occurring in an eight-year-old child: a case report. J Bone Joint Surg Am 48:946-948

4. Patel K et al. (2006) Epidermal inclusion cyst of the phalynx: a case report and review of the literature. Skeletal Radiol 35:861-863

5. Roth SI (1964) Squamous cysts involving the skull and distal phalanges. J Bone Joint Surg Am 46:1442-1450

6. Schajowicz F, Aiello CL, Slullitel I (1970) Cystic and pseudocystic lesions of the terminal phalynx with special reference to epidermoid cysts. Clin Orthop Relat Res 68:84-92

7. Wang BY et al. (2003) Intraosseous epidermoid inclusion cyst in a great toe: a case report and review of the literature. Arch Pathol Lab Med 127:298-300 